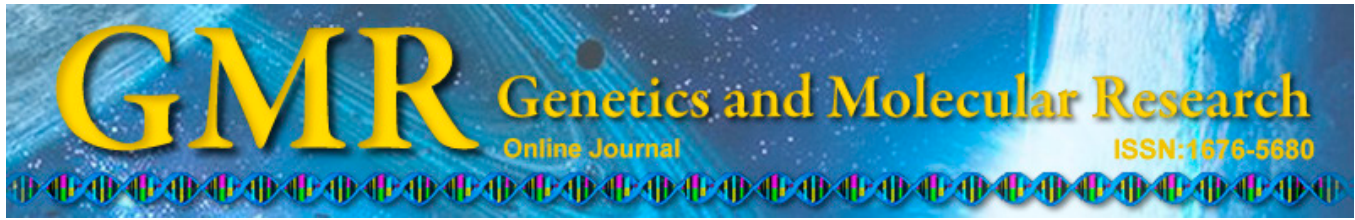

\title{
S100A4 expression and prognosis of gastric cancer: a meta-analysis
}

\author{
Y. Wang ${ }^{1 *}$, L.-B. Zhou ${ }^{1 *}$ and X.-H. Li ${ }^{2}$ \\ ${ }^{1}$ Department of General Surgery, \\ Second Affiliated Hospital of Anhui Medical University, Hefei, China \\ ${ }^{2}$ Department of Digestive Medicine, Shanghai Eighth People's Hospital, \\ Shanghai, China \\ *These authors contributed equally to this study. \\ Corresponding author: X.-H. Li \\ E-mail: lixinghua212@yeah.net
}

Genet. Mol. Res. 13 (4): 10398-10403 (2014)

Received October 9, 2013

Accepted July 26, 2014

Published December 12, 2014

DOI http://dx.doi.org/10.4238/2014.December.12.1

\begin{abstract}
The prognostic role of S100A4 in gastric cancer is still under debate. The present meta-analysis aimed to evaluate the relationship between S10A4 levels and the prognosis of gastric cancer. We performed a meta-analysis of published studies assessing the relationship between $\mathrm{S} 100 \mathrm{~A} 4$ and gastric cancer prognosis. We used the Revman 5.0 software to perform literature retrieval, article selection, data collection, and statistical analysis. A fixed-effect model was used to pool the hazard ratio (HR) and $95 \%$ confidence intervals $(95 \% \mathrm{CI})$. A total of 7 eligible studies that included 1257 gastric cancer patients were analyzed. We did not find a prognostic value for S100A4 in gastric cancer $(\mathrm{HR}=1.48,95 \% \mathrm{CI}=0.77$ to $2.82, \mathrm{P}=0.24)$. In conclusion, the present study indicated that $\mathrm{S} 100 \mathrm{~A} 4$ expression level is not a prognostic factor for gastric cancer.
\end{abstract}

Key words: S100A4; Prognosis; Gastric cancer; Meta-analysis 


\section{INTRODUCTION}

Gastric cancer is one of the most common causes of cancer-related death in recent years (El-Rifai and Powell, 2002). Worldwide, approximately 876,000 people are diagnosed with this disease every year and approximately 649,000 succumb (Durrani et al., 2009). In China, there are approximately 160,000 deaths from gastric cancer annually (Chen et al., 2004). A number of studies have reported the relationship between S100A4 expression and the prognosis of gastric cancer (Yonemura et al., 2000; Yu et al., 2006; Huang et al., 2008; Wang et al., 2010; Feng et al., 2011; Stein et al., 2011; Zhao et al., 2013; Li et al., 2013) However, the association of S100A4 with gastric prognosis remains unclear.

The S100 protein family was first isolated from bovine brain by Moore (1965). Subsequent studies identified 16 members of this family, based on amino acid sequence homology and similar structural properties (Schafer and Heizmann, 1996). S100A4 is highly expressed in many cancers (Kikuchi et al., 2006; Ai et al., 2008; Kwak et al., 2010) and its presence predicts a poor outcome, such as in pancreatic cancer, (Ai et al. 2008), colorectal cancer (Kwak et al., 2010), ovarian carcinoma (Kikuchi et al., 2006), bladder carcinoma (Yao et al. 2007), esophageal carcinoma (Ji et al., 2004), and gastric carcinoma (Yonemura et al., 2000; Yu et al., 2006; Huang et al., 2008; Feng et al., 2011; Wang et al., 2010; Stein et al., 2011; Zhao et al., 2013; Li et al., 2013). However, the prognostic value of S100A4 in patients with gastric cancer is still controversial. This study was a retrospective analysis of published studies to evaluate the prognostic value of S100A4 level before treatment in patients with gastric cancer.

\section{MATERIAL AND METHODS}

\section{Publication search}

We searched the PubMed, Medline, Embase, AACR (American Association for Cancer Research), Chinese Biomedical Literature Database, China National Knowledge Infrastructure (CNKI), and Wanfang databases using the search terms "S100A4" and "gastric cancer" or "gastric carcinoma" and "prognosis" updated until July 2013. The online search was accompanied by checking reference lists from the articles and reviews identified for potentially eligible original reports.

\section{Inclusion criteria}

The inclusion criteria were as follows: 1) clinical research on direct comparison of S100A4 levels in gastric cancer before and after treatment, without any restriction on language or publication year; 2) research subjects were gastric cancer patients without any restriction on age or race; 3) outcome indicators: overall survival.

\section{Exclusion criteria}

The major exclusion criteria were as follows: 1) duplicate data; 2) case reports, series, abstract, comment, review, and editorial; 3) insufficient data. 


\section{Literature quality assessment and data extraction}

We collected the information as follows: author, year of publication, country of origin, ethnicity, number of cases, and S100A4 detection method. In a few studies, part of the data had already been reported elsewhere, therefore, only the novel data was included.

\section{Data analysis}

Meta-analysis was performed by using the RevMan 5.0 software provided by the Cochrane Collaboration. We directly used the q-test and the $\mathrm{I}^{2}$ test to examine the heterogeneity between each study. We used the hazard ratio (HR) value to evaluate the relationship between the S100A4 level and overall survival in gastric cancer. To test for publication bias, we used the RevMan 5.0 statistical software to make the funnel plot. $\mathrm{P}<0.05$ was considered as indicating a significant difference.

\section{RESULTS}

\section{Literature screening}

A total of 104 articles were initially identified, 97 articles were excluded because of duplicate publication and non-clinical research study. A total of 7 articles were included, of which all were clinical studies, with four belonging to the Chinese literature and three to English literature (Table 1). The 7 studies including 1257 patients were included in this research.

\begin{tabular}{|c|c|c|c|c|}
\hline First Author & Publication year & Country & HR & $95 \% \mathrm{CI}$ \\
\hline $\mathrm{Li}$ & 2013 & China & 10.129 & $4.56-21.43$ \\
\hline Feng & 2011 & China & 3.233 & $1.04-5.42$ \\
\hline Stein & 2011 & Germany & 2.76 & $1.03-4.98$ \\
\hline Huang & 2008 & China & 2.126 & $1.10-4.099$ \\
\hline $\mathrm{Yu}$ & 2006 & China & 3.12 & $2.11-6.33$ \\
\hline Wang & 2010 & China & 2.19 & $1.33-3.55$ \\
\hline Yonemura & 2000 & Switzerland & 2.43 & $1.32-4.35$ \\
\hline
\end{tabular}

\section{S100A4 level and prognosis for gastric cancer}

In 3 of these 7 studies, we extracted the HR values and their 95\% CI directly and used them for the evaluation of the association of S100A4 levels with the prognosis of gastric cancer. In the other four studies, the HR values and their $95 \% \mathrm{CI}$ could be calculated according to the data. There was better homogeneity between each study $\left(\mathrm{P}=1.00, \mathrm{I}^{2}=0 \%\right)$. We did not find a prognostic value for $\mathrm{S} 100 \mathrm{~A} 4$ in gastric cancer. $(\mathrm{HR}=1.48,95 \% \mathrm{CI}=0.77$ to 2.82 , $\mathrm{P}=0.24 ;$ Figure 1).

\section{Publication bias analysis}

We analyzed publication bias by use of the Revman 5.0 software. The funnel plot (Figure 2) showed the points evenly distributed, and most of the points were within the $95 \% \mathrm{CI}$. It indicated that there was no publication bias, and thus, the results of the study were credible. 


\begin{tabular}{|c|c|c|c|c|c|}
\hline Studv or Subaroup & log[0dds Ratio] & SE & Weight & $\begin{array}{l}\text { Odds Ratio } \\
\text { IV, Fixed, } 95 \% \mathrm{Cl}\end{array}$ & $\begin{array}{c}\text { Odds Ratio } \\
\text { IV, Fixed, } 95 \% \mathrm{Cl}\end{array}$ \\
\hline Feng 2011 & 0.51 & 1.12 & $8.7 \%$ & $1.67[0.19,14.96]$ & \\
\hline Huang 2008 & 0.33 & 0.76 & $18.8 \%$ & $1.39[0.31,6.17]$ & \\
\hline Li 2013 & 1.01 & 4.3 & $0.6 \%$ & $2.75[0.00,12554.63]$ & \\
\hline Wang 2010 & 0.34 & 0.57 & $33.5 \%$ & $1.40[0.46,4.29]$ & \\
\hline Stein 2011 & 0.44 & 1.01 & $10.7 \%$ & $1.55[0.21,11.24]$ & \\
\hline Yonemura 2000 & 0.39 & 0.77 & $18.4 \%$ & $1.48[0.33,6.68]$ & \\
\hline Yu 2006 & 0.49 & 1.08 & $93 \%$ & $1.63[0.20,13.56]$ & \\
\hline Total $(95 \% \mathrm{Cl})$ & & & $100.0 \%$ & $1.48[0.77,2.82]$ & \\
\hline \multicolumn{4}{|c|}{$\begin{array}{l}\text { Heterogeneity }\left.C h\right|^{2}=0.06, d f=6(P=1.00) ;\left.\right|^{z}=0 \% \\
\text { Test for owerall effect: } Z=1.18(P=0.24)\end{array}$} & & $\begin{array}{lll}0.01 & 0.1 & 1 \\
& \text { Favors [High] }\end{array}$ \\
\hline
\end{tabular}

Figure 1. Forest plot of prognosis of gastric cancer and S100A4 levels. The horizontal lines correspond to the study-specific OR and $95 \% \mathrm{CI}$, respectively. The area of the squares reflects the study-specific weight. The diamond represents the pooled results of OR and 95\%CI. In this analysis, a fixed-effect model was used.

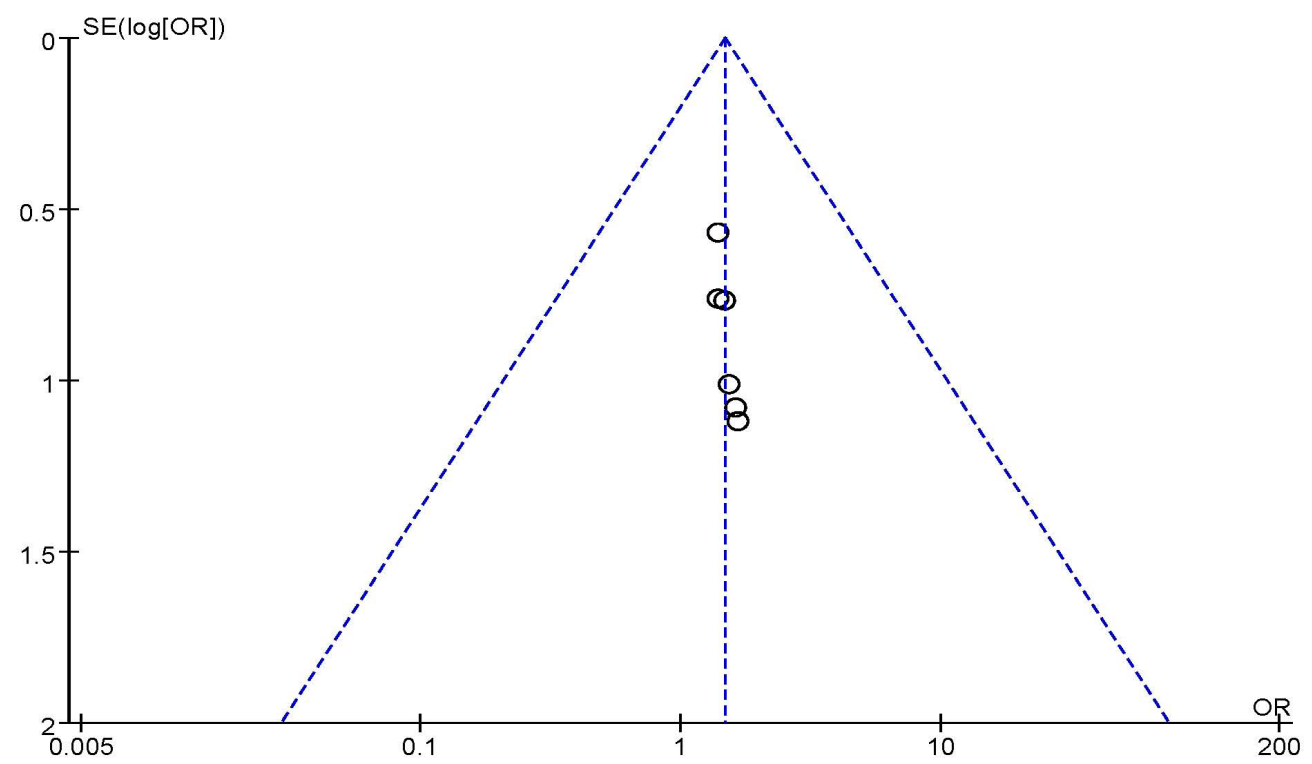

Figure 2. Begg's funnel plot for publication bias test. Each point represents a separate study for the indicated association. Log OR represents natural logarithm of OR. Vertical line represents the mean effect size.

\section{DISCUSSION}

In the present study, we found that S100A4 was not associated with the prognosis of gastric cancer by a meta-analysis. 
The S100A4 protein is one of 24 members of the S100 family. The S100 proteins regulate the interaction between $\mathrm{Ca}^{2+}$ and target proteins. S100A4 is involved in the regulation of a wide range of intracellular and extracellular biological effects, including cell motility, angiogenesis, cell survival, differentiation, contractility and invasion (Stein et al., 2006). Studies have indicated that S100A4 is associated with tumor invasion and metastasis, and that it may be a potential prognostic marker. Kwak et al. (2010) detected immunoreactivity for S100A4 in $45(35.4 \%)$ of 127 colorectal cancers and found that S100A4 protein expression was associated with tumor recurrence and poor overall survival in patients with colorectal cancer. Kikuchi et al. (2006) demonstrated that nuclear expression of S100A4 was involved in the aggressive behavior of ovarian carcinoma and that S100A4 is an autocrine/paracrine factor that plays an important role in the aggressiveness of ovarian carcinoma cells. However, the association of S100A4 with gastric cancer remains controversial. In the present study, we performed a meta-analysis including 1257 patients with gastric cancer and did not find any association of S100A4 concentration with poor prognosis of gastric cancer. The seven studies included in this meta-analysis had clear diagnostic, inclusion and exclusion criteria. The patients were grouped according to S100A4 levels, and the overall survival was the main outcome. The HR value was a statistical indicator to assess the impact of different levels of S100A4 on overall survival of patients with gastric cancer. Also, the present study included not only the English literature, but also the Chinese literature for which the full texts could be provided and the summaries of the unpublished studies were excluded. However, this method might have caused selection bias.

In summary, this meta-analysis of 7 studies showed that the S100A4 level is not of prognostic significance in patients with gastric cancer.

\section{REFERENCES}

Ai KX, Lu LY, Huang XY, Chen W, et al. (2008). Prognostic significance of S100A4 and vascular endothelial growth factor expression in pancreatic cancer. World J. Gastroenterol. 14: 1931-1935.

Chen XM, Chen GY, Wang ZR, Zhu FS, et al. (2004). Detection of micrometastasis of gastric carcinoma in peripheral blood circulation. World J. Gastroenterol. 10: 804-808.

Durrani AA, Nayyar Y, Shahid A, Masood S, et al. (2009). Pattern of upper gastrointestinal malignancies in Northern Punjab. Pak. J. Med. Sci. 25: 302-307.

El-Rifai W and Powell SM (2002). Molecular biology of gastric cancer. Semin. Radiat. Oncol. 12: 128-140.

Feng LZ, Zheng XY, Zhou LX, Fu B, et al. (2011). Correlation between expression of S100A4 and VEGF-C, and lymph node metastasis and prognosis in gastric carcinoma. J. Int. Med. Res. 39: 1333-1343.

Huang HL, Wu BY, Zhu XD, et al. (2008). Studies on correlation between S100A4 expression and biological behavior and prognosis of gastric cancer. Med. J. Chin. PLA 33: 506-508.

Ji J, Zhao L, Wang X, Zhou C, et al. (2004). Differential expression of S100 gene family in human esophageal squamous cell carcinoma. J. Cancer Res. Clin. Oncol. 130: 480-486.

Kikuchi N, Horiuchi A, Osada R, Imai T, et al. (2006). Nuclear expression of S100A4 is associated with aggressive behavior of epithelial ovarian carcinoma: an important autocrine/paracrine factor in tumor progression. Cancer Sci. 97: 1061-1069.

Kwak JM, Lee HJ, Kim SH, Kim HK, et al. (2010). Expression of protein S100A4 is a predictor of recurrence in colorectal cancer. World J. Gastroenterol. 16: 3897-3904.

Li H, Liu Z, Xu C, Chen Y, et al. (2013). Overexpression of S100A4 is closely associated with the progression and prognosis of gastric cancer in young patients. Oncol. Lett. 5: 1485-1490.

Moore BW (1965). A soluble protein characteristic of the nervous system. Biochem. Biophys. Res. Commun. 19: 739-744.

Schafer BW and Heizmann CW (1996). The S100 family of EF-hand calcium-binding proteins: functions and pathology. Trends Biochem. Sci. 21: 134-140.

Stein U, Arlt F, Walther W, Smith J, et al. (2006). The metastasis-associated gene S100A4 is a novel target of beta- 
catenin/T-cell factor signaling in colon cancer. Gastroenterology 131: 1486-1500.

Stein U, Burock S, Herrmann P, Wendler I, et al. (2011). Diagnostic and prognostic value of metastasis inducer S100A4 transcripts in plasma of colon, rectal, and gastric cancer patients. J. Mol. Diagn. 13: 189-198.

Wang YY, Ye ZY, Zhao ZS, Tao HQ, et al. (2010). High-level expression of S100A4 is correlated with lymph node metastasis and poor prognosis in patients with gastric cancer. An. Surg. Oncol. 17: 89-97.

Yao R, Lopez-Beltran A, Maclennan GT, Montironi R, et al. (2007). Expression of S100 protein family members in the pathogenesis of bladder tumors. Anticancer Res. 27: 3051-3058.

Yonemura Y, Endou Y, Kimura K, Fushida S, et al. (2000). Inverse expression of S100A4 and E-cadherin is associated with metastatic potential in gastric cancer. Clin. Cancer Res. 6: 4234-4242.

Yu GZ, Wang JJ, Chen Y and Ni CR (2006). Expression of KISSI and S100A4 in gastric carcinoma and the correlation with metastasis. Tumor 26: 721-724.

Zhao Y, Zhang T and Wang Q (2013). S100 calcium-binding protein A4 is a novel independent prognostic factor for the poor prognosis of gastric carcinomas. Oncol. Rep. 30: 111-118. 